\title{
Prescribing placebos: an experimental examination of the role of dose, expectancies, and adherence in open-label placebo effects
}

\begin{abstract}
Background: Recent evidence indicates that placebo effects can occur even when patients know that they are taking a placebo, termed the open-label placebo effect.

Aim: To assess whether placebo dose (1 pill per day versus 4 pills per day), treatment expectancies, and adherence contribute to open-label placebo effects.

Method: Healthy undergraduate participants were randomly assigned to take 1 or 4 openlabel placebo pills per day, or to a no treatment control group. Placebo-treated participants took a 5-day course of an open-label placebo described as enhancing physical (symptoms and sleep) and psychological (positive and negative emotional experience) wellbeing. Expectancies about placebo effectiveness and wellbeing were assessed at baseline, and wellbeing and adherence were assessed after the 5-day course of treatment.

Results: Medium to large open-label placebo effects were evidenced in all wellbeing outcomes including sleep quality. Dose did not influence these effects. Both treatment expectancies and adherence were significant independent predictors of enhanced wellbeing in the two psychological wellbeing outcomes and the experience of physical symptoms but sleep quality improved independently.

Conclusions: This the first study to demonstrate the effect of open-label placebos in improving wellbeing and sleep quality, and to show that open-label placebo reposes do not appear to be dose-dependent, but for most wellbeing outcomes are independently predicted by both positive expectancies and treatment adherence.
\end{abstract}




\section{INTRODUCTION}

Placebo effects are the beneficial or healing effects of an inert treatment which result from receiving the treatment, but are not caused by any active ingredient in the treatment [1]. Time and time again, the placebo effect has been shown to have a clinically significant impact on not only subjective health outcomes but also objective physiological measures for a variety of disease states $[2,3]$. The same inert sugar pill can induce the release of endogenous opioids for analgesia [4,5], increase cortical glucose metabolism in those with depression [6], or even increase the release of dopamine in the brains of patients with Parkinson's Disease [7], simply by altering the treatment information and delivery context. Harnessing placebo effects offers the possibility of improving patient outcomes and enhancing the overall efficacy of active medical treatments.

One of the primary hurdles to the clinical implementation of placebo treatments are the ethical issues relating to use of deception, a feature common to many placebo treatments. It is often considered necessary for patients to believe (and thus be deceived) that they are taking a pharmacologically active treatment in order to harness the placebo effect $[2,8]$. The use of deception in clinical care has the potential to cause harm to individuals if they feel their autonomy has not been respected, and may also weaken patients' trust in the medical profession [9]. As such, the perceived need for deception when administering placebos presents a barrier to widespread utilisation of placebo effects in medical care.

Recent research, however, has demonstrated that placebos can still induce clinically significant results without the use of deception, termed the 'open-label placebo'. Open-label placebos, like all placebos, are inert treatments containing no active ingredient. The key difference is that these placebos are given to patients alongside the knowledge that the pill is an inert placebo. Strikingly, open-label placebo treatments have been shown to be efficacious 
across a range of conditions and patients, including children with ADHD [10], chronic low back pain and migraine pain patients $[11,12]$, and can also significantly improve symptoms and quality of life in patients with Irritable Bowel Syndrome [13]. There are also preliminary findings of a therapeutic effect of open-label placebos in depression [14]. These results defy the conventional wisdom that placebos only work when patients believe they are active treatments, and provide the preliminary support for an ethical implementation of the placebo effect in clinical practice.

Very little is currently known about the mechanisms by which open-label placebos have their effect [15]. However, much more is understood about placebo effects more generally from research using deceptive placebo paradigms. Two primary interrelated mechanisms have been supported as underlying placebo effects: expectancies, and classical conditioning [1]. Expectancy theory proposes that anticipating a particular outcome (e.g. symptom relief) can lead to that outcome being realised [16]. Classical conditioning of placebo effects involves the repeated pairing of the treatment context (e.g., a particular pill or salient drink with which a tablet is always taken) with the beneficial effects on an active ingredient. Subsequent exposure to the treatment context alone - which previously had no effect - results in similar effects to that of the active ingredient [17]. This direct experience of treatment benefit through a classical conditioning process can also result in positive expectancies, which mediate many conditioned placebo effects $[1,18]$. Conditioned placebo effects can persist following disclosure that the treatment was a placebo [19], but this still requires deception in the initial conditioning procedure. The perception that a given treatment is ineffective blocks the formation conditioned placebo effects [20], suggesting that prior conditioning does not provide an adequate explanation for the existence of open-label placebo effects. Although expectancies provide a promising explanation for the effectiveness of open-label placebo treatments, this has not yet been assessed. 
Another important consideration for the use of open-label placebos in clinical practice is the 'dose' that should be prescribed. Prescription medications come with clear instructions regarding dosage, including how and when to take the treatment, which are determined by the pharmacological and pharmacokinetic properties of the drug, as well as clinical characteristics of the patient. Placebo treatments contain no active ingredients, thus the number of pills prescribed and dosing regimen cannot be determined in the same way. In studies experimentally investigating the effectiveness of open-label placebos, patients have typically been prescribed four placebo pills: two pills twice per day $[11,13,14]$. This is in line with previous evidence indicating that taking more placebo pills produces larger effects $[21,22]$.

In contrast, simplified dosing regimens comprising fewer daily doses have been shown to substantially increase treatment adherence [23]. Patients who are highly adherent to placebo treatments in RCTs have better health outcomes, compared to less adherent patients [24]. Effects have been found in patients treated for heart failure, myocardial infarction, HIV, type 2 diabetes, and immunosuppression [25]. Good adherence to placebo treatment was associated with about half the mortality risk of poor adherence. Although this decreased mortality is commonly attributed to a "healthy adherer effect" - whereby highly adherent patients have better outcomes because they are also likely to engage in other beneficial health behaviours - enhanced placebo effects or an enhanced placebo component of active treatments may also contribute to these beneficial outcomes [26]. The current standard openlabel placebo dosing schedule (two placebo pills taken twice per day), and the influence of adherence on placebo effects, are at odds with one another. On one hand, we might expect that taking more placebo pills more frequently might enhance the placebo effect. On the other, it may be that more simple dosing regimens encourage higher adherence, and thus larger placebo effects. 
One way to assess the mechanisms underlying the open-label placebo effect is to develop relevant experimental paradigms using non-clinical samples. Numerous studies have utilised non-clinical samples to investigate the 'deceptive' placebo effect, using diverse research paradigms including the alleviation of pain, itch, insomnia, nausea, and anxiety [e.g. 26-31]. Such paradigms have allowed experimental assessment of underlying mechanisms contributing to the placebo effect, in a way that is more challenging to achieve in clinical populations. To date, experimental assessment of open-label placebo effects has been in patient groups only, including irritable bowel syndrome [13], major depressive disorder [14], and chronic low back pain [11].

The current study explores the possibility that healthy participants also experience open-label placebo effects, and tests a novel paradigm in which the effect of the open-label placebo is described as enhancing physical and psychological wellbeing. The concept of wellbeing is central to health, which defined by the World Health Organisation as "a state of complete physical, mental, and social wellbeing" [33]. Improved biomarkers of illness without concurrent improvements in subjective wellbeing are unlikely to be deemed successful or even adequate by patients [34].

Physical wellbeing is assessed as the experience of physical symptoms, and sleep quality. The experience of physical symptoms is an integral part of how people view and manage their health [35], and the experience of symptoms is a primary driver of seeking medical care [36]. Poor sleep can be a precursor to or comorbid with chronic illness, and is associated with outcomes such as elevated blood pressure in young adults [37], and increased risk of developing type 2 diabetes [38]. Psychological wellbeing comprised negative and positive emotional states. The experience of negative emotions - including depression, anxiety, and stress - can both cause and result from chronic ill health [39]. For example, the experience of depression and anxiety predict the development of coronary heart disease and 
type 2 diabetes [40,41]. Stress is consistently linked with heart disease, poor wound healing, and impaired immune function [42,43]. In contrast, the experience of positive emotions (independent of distress) has been linked to improved neuroendocrine, cardiovascular, and inflammatory activity [44], as well as longer-term health benefits including increased longevity, enhanced immune function, and reduced burden of heart disease [45-48].

As such, although the paradigm was designed to investigate open-label placebo effects in healthy participants, the chosen outcomes are applicable and important to health behaviours and health outcomes in patient populations. Improvements in symptoms, quality of life, and depression have also been identified in open-label placebo research with patient populations $[13,14]$. Developing experimental open-label placebo paradigms with healthy participants will enable further exploration of the mechanisms underlying these effects. The aim of the current study is to empirically test the influence of expectancies, dose, and adherence, on open-label placebo effects on physical and psychological wellbeing in healthy participants. Investigating the impact of these factors is an important step in enhancing understanding of the open-label placebo effect, and utilising non-deceptive placebo treatments in clinical practice.

\section{METHODS}

Participants were recruited to take part in research investigating the effectiveness of open-label placebo administration on wellbeing. Four wellbeing factors were assessed: positive mental wellbeing, negative emotional states (depression, anxiety, and stress), physical symptoms, and sleep quality. In all advertisements, the participant information statement, and the baseline study session, participants were informed that they would receive inert placebo capsules containing only lactose (described as 'sugar pills'). Participants were excluded if they were unable to consume lactose. The research was approved by the UNSW Human Research Ethics 
Advisory Panel C (Behavioural Sciences; file 2770), and was pre-registered with the Australia and New Zealand Clinical Trials Registry (ACTRN12617000253303).

\section{Design}

This study used a between-subjects experimental design, with participants randomly assigned to one of three possible treatment conditions: 1) a no treatment control condition, 2) one placebo pill-per-day, or 3) four placebo pills-per-day. Baseline assessments of wellbeing, expectancies, and demographics were carried out during an in-person study session with the male experimenter, who was blind to treatment condition during this assessment, and participants completed these questionnaires on a computer running Qualtrics software in a research cubicle while the experimenter was in another room. Participants in the placebo conditions were then given placebo capsules to take home with them to take over the next five days. After this, participants completed online follow-up questionnaires comprising the same wellbeing measures as at baseline, as well as self-reported adherence.

\section{Participants}

Potential participants were recruited from the undergraduate psychology participant pool at the University of New South Wales, and received course credit for their participation. In total, 92 participants were enrolled in the study, provided with information about open-label placebos, completed the baseline assessments, and randomised to one of the three treatment conditions in order of attendance according to a pre-determined schedule using the random number generator in Excel. The experimenter was blind to group allocation until participants had completed the baseline questionnaires. Group allocation was approximately equal: 30 participants were assigned to the no treatment control condition, 31 to the 1 placebo-per-day condition, and 31 to the 4 placebos-per-day condition. In the no treatment control group, 27 participants (90\% retention) completed follow-up questionnaires, as did 30 participants $(97 \%)$ in the 1 placebo-per-day condition, and 31 participants (100\%) in the 4 placebos-per- 
day condition. The mean age of the sample was 19 years $(S D=3.9$; range 18 to 44$)$, and $80 \%$ were female.

\section{Materials}

\section{Placebo Capsules}

The placebos in this study were clear plant-based gel capsules containing lactose powder. Participants receiving placebos were given an amber pill bottle with a white screw cap containing their placebo capsules ( 1 or 4 placebo pills to take per day) to take away with them. These bottles were labelled with branding specifically designed for this study by the first author (JEB), and included the brand name ('Plaxibax'), description of the contents, and dosing instructions (see Figure 1). We chose to create a brand name and associated labelling for the open-label placebos because previous research indicates that an association with branding $[49,50]$ can increase 'deceptive' placebo effects.

\section{Measures}

At both baseline and at follow-up, participants were asked to complete questionnaires assessing different aspects of psychological and physical wellbeing.

Emotional distress: the Depression Anxiety Stress Scale 21 (DASS-21) is a 21-item self-report questionnaire comprising depression, anxiety, and stress subscales [48]. Participants respond to items (e.g. "I couldn't seem to experience any positive feeling at all") on a 4-point scale from 0 (never) to 3 (almost always). Total scores were calculated by summing responses to all items, ranging from 0 (very low) to 63 (extremely severe) emotional distress. The DASS-21 has excellent internal consistency (Cronbach's alpha $=$ .93), and demonstrates good convergent validity with other validated measures of depression and anxiety [52].

Positive emotions: the Warwick-Edinburgh Mental Wellbeing Scale (WEMWBS) is a 14-item self-report questionnaire that assesses overall positive mental wellbeing [53]. 
Responses to items (e.g. "I have been feeling good about myself”) are reported on a 5-point Likert scale from 1 (none of the time) to 5 (all of the time), with total scores from 14 to 70 , and higher scores indicating more positive mental wellbeing. This scale shows little evidence of floor or ceiling effects in either student or general populations. The WEMWBS demonstrates good to excellent internal consistency (Cronbach's alpha $=.89$ to .91 ), as well as good content, convergent, and discriminant validity, and high test-retest reliability [53].

Physical symptoms: symptoms were assessed using the Subjective Health Complaints inventory (SHC), a list of 29 symptoms (e.g. headache, dizziness, diarrhoea) on which individuals rate the severity of each symptom over the past week [54]. The scale was modified slightly for the current study to ask about symptoms over the past 5 days. Each symptom is rated on an intensity scale from 0 (not at all) to 3 (severe), and these ratings are summed to give a score ranging from 0 to 87 . The SHC has acceptable to good internal consistency (Cronbach's alpha $=.75$ to .82 ) [54], and high scores on this scale are associated with increased healthcare utilisation [55].

Sleep quality: the Pittsburgh Sleep Quality Index (PSQI) is a self-report questionnaire assessing sleep quality [56]. The scale has high validity and reliability and is the most widely used standardised measure of sleep quality [57]. The PSQI assesses sleep quality for the previous month (e.g. "during the past month, how often have you had trouble sleeping because you wake up in the middle of the night or early morning?"). To make the scale appropriate for the timeframe of the current study a modified version was used to assess sleep quality over the past 5 days. The scale comprises seven components - each scored from 0 to 3 - which are summed to provide a Global PSQI score ranging from 0 (very good sleep) to 21 (very bad sleep). Total scores of 5 or higher indicate poor sleep. The PSQI shows good internal consistency (Cronbach's alpha $=.80)$, and discriminant and convergent validity [58]. 
Expectancies: in addition to the wellbeing questionnaires, after being provided with information about the open-label placebo effect, participants were asked about their expectancies of how well an open label placebo ('Plaxibax') would work to enhance their wellbeing on a scale from 0 (not at all) to 10 (extremely well). Question wording was as follows: "we would like to ask about your expectations regarding the effect of taking placebo pills on your general wellbeing. How well do you think the placebo pills will work for you?" Adherence: at follow-up, participants again completed the wellbeing questionnaires, and placebo-treated participants were also subsequently asked about how well they had adhered to the placebo treatment ("Over the past 5 days you were instructed to take 1 (or 4 ) placebo pill(s) per day; how well did you follow these instructions?") on a scale from 0 (not well at all) to 10 (extremely well). Participants were also asked to provide a numerical response to the question "approximately how many placebo pills have you missed over the past 5 days?" These questions were prefaced with a statement asking participants to be honest, noting that there would be no repercussions for non-adherence, and explaining the importance of accurate information for the researchers.

Because the two placebo-treated groups were given different numbers of pills to take per day, the number of missed pills was not directly comparable between the two groups, and this variable was not used in subsequent analyses. However, there was a significant negative correlation between self-reported adherence and number of missed pills, $r(60)=-.48$. Only one participant reported missing more than $20 \%$ of their placebo pills (i.e. taking less than $80 \%$ of doses). In clinical studies, treatment adherence of $80 \%$ or more is associated with better clinical outcomes $[59,60]$, and this is a commonly accepted level to determine clinical adherence. However, this participant also rated themselves as a 9 out of 10 in following their treatment instructions, suggesting that they may have misinterpreted the question as asking 
about how many pills they had taken, rather than how many they had missed. Based on these two adherence items, participants can be considered as adherent to their placebo pills.

Demographics: at baseline participants were asked about their age (in years) and gender (male, female, or other).

\section{Procedure}

Study participation took place over seven days. On day 1, participants attended a 30-minute one-on-one face-to-face session with a researcher in which information about placebos was provided, and baseline questionnaires were administered. The experimenter first explained clearly that the placebo pills were inert, with no active ingredient, 'like sugar pills'. This information was followed by four discussion points outlined by Kaptchuk and colleagues [13]. Briefly, these were: 1) the placebo effect is powerful and placebos have been shown in numerous clinical trials to generate real physiological effects, 2) your body can automatically respond to taking placebo pills to activate mind-body healing processes, 3) positive expectations can help but are not crucial, and 4) that taking the pills as prescribed is important.

Following the placebo information provision, participants completed a baseline questionnaire, and were informed of their group allocation. Participants randomised to a placebo condition were also given placebo pills to take home with them, and instructions about how to take them over the next six days. Participants in the 1 placebo-per-day condition were given six placebo capsules and were instructed to take one each morning. Participants in the 4 placebos-per-day condition received 24 capsules with instructions to take two in the morning and two at night. On day 2, participants began their placebo treatment. On day 4 (the third day of treatment), participants were sent a reminder email. All participants were thanked for attending the baseline session, and reminded that they would receive an email with a link to the follow-up questionnaire on day 7. In the placebo conditions, participants were also 
reminded to take the placebo capsules as instructed. On day 7 , participants received a link to the online follow-up questionnaire.

\section{Statistical Analyses}

All analyses were carried out using SPSS v.23. First, analysis of variance (ANOVA) and chisquare analyses were used to test for any differences across the three groups in demographic and wellbeing factors reported at baseline.

Next, analysis of covariance (ANCOVA) was used to assess the influence of treatment condition (no treatment control, one placebo per day, four placebos per day) on wellbeing outcomes (depression, anxiety, and stress scores, positive mental wellbeing, symptom burden, and sleep quality) while controlling for the baseline scores of the outcome variable under consideration, as per Vickers and Altman (2001). Separate analyses were carried out for each outcome. Planned orthogonal contrasts were used, in which 1) the control group was compared to the mean of the two placebo-treated groups to test for an open-label placebo effect, and 2) the 1 placebo-per-day group was compared to the 4 placebos-per-day group to test for a dose effect.

Finally, exploratory multiple linear regression analyses (not pre-registered) were conducted to examine the influence of expectancies and treatment adherence on open-label placebo effects. Separate analyses were carried out for each wellbeing outcome. Because these analyses assessed the role of adherence, only placebo-treated participants were included. Variables were entered in three steps. In the first step, baseline scores of the outcome variable as well as dose condition were entered. In the second step, expectancy ratings and self-reported treatment adherence were entered. The interaction term between baseline expectancy and self-reported adherence was entered in the third step. An alpha level of 0.05 was used for all tests.

\section{RESULTS}


At baseline, there were no differences between the groups in gender or age (see Table 1). Participants also did not differ in the experience of emotional distress, positive mental wellbeing, symptom scores, or sleep quality. Similarly, participants did not differ by group on baseline expectancies for treatment effectiveness (all participants received identical information and completed this measure prior to randomisation).

Paired samples t-tests were conducted to assess whether baseline and follow-up wellbeing scores of participants in the no-treatment control group differed significantly. There were no significant differences over time for emotional distress $(p=.88)$, positive emotional wellbeing $(p=.07)$, physical symptoms $(p=.83)$, or sleep quality $(p=.54)$.

\section{Open-Label Placebo Effects}

Emotional distress: participants who had taken the open-label placebo capsules experienced a substantial placebo effect as evidenced by significantly lower DASS scores at follow-up, compared to the no treatment control participants, $F(1,84)=13.94, p<.001, \eta_{p}^{2}=$ .14 (see Figure 2A and Table 2). On average, the placebo-treated participants reported DASS scores that were 5.56 points lower than participants in the control condition (scale range: 0 $63 ; S E=1.49,95 \% \mathrm{CI}[-8.52,-2.60])$. The emotional distress scores of the 1 placebo-per-day and 4 placebos-per-day groups were not significantly different from one another, $F(1,84)=$ $1.32, p=.25, \eta_{p}^{2}=.02$

Positive mental wellbeing: there was evidence of an open label placebo effect, with placebo-treated participants reporting enhanced positive mental wellbeing compared to those in the no treatment control condition, $F(1,84)=10.28, p=.002, \eta_{p}^{2}=.11$ (see Figure $2 \mathrm{~B}$ and Table 2). Participants in who took the open-label placebo treatment had WEMWBS scores that were, on average, 5.84 points higher than participants in the control condition (scale range: $14-70 ; S E=1.82,95 \% \mathrm{CI}[2.22,9.46])$. The positive mental wellbeing scores of the 1 
placebo-per-day and 4 placebos-per-day groups did not differ significantly, $F(1,84)=2.28, p$ $=.14, \eta_{p}^{2}=.03$.

Physical symptoms: the planned contrast between the control group and the placebo treated groups revealed a significant placebo effect on symptoms, $F(1,83)=4.73, p=.032$, $\eta_{p}^{2}=.05$ (see Figure 2C and Table 2). Placebo-treated participants reported symptoms scores that were, on average, 5.95 points lower than those in the no treatment control group (scale range: $0-87 ; S E=2.73,95 \% \mathrm{CI}[-11.39,-0.51])$. The 1 placebo-per-day and 4 placebos-perday groups did not differ in their physical symptoms, $F(1,83)=1.95 p=.90, \eta_{p}^{2}<.001$.

Sleep quality: overall, there was an open-label placebo effect on sleep quality when comparing the control and placebo treated participants, $F(1,83)=13.95, p<.001, \eta_{p}^{2}=.14$ (see Figure 2D and Table 2). Those in the open-label placebo group had PSQI scores that were, on average, 1.57 points lower (indicating improved sleep quality) than participants in the control condition (scale range: 0 to $21 ; S E=0.42,95 \% \mathrm{CI}[-2.40,-0.73]$ ). The 1 placeboper-day and 4 placebos-per-day groups did not differ significantly in their reported sleep quality, $F(1,83)=1.82, p=.18, \eta_{p}^{2}=.02$.

\section{Predictors of Open-Label Placebo Effects}

Next, we tested whether treatment expectancies and self-reported adherence were predictive of open-label placebo effects in each of the four wellbeing outcomes. These analyses were carried out using data from only placebo-treated participants who completed the follow-up questionnaire.

Expectancies and adherence: expectancy ratings at baseline did not differ between the 1 placebo-per-day $(M=5.13, S E=0.44)$ and 4 placebos-per-day $(M=5.00, S E=0.40)$ groups, $t(60)=0.22, p=.83$. Self-rated adherence to the placebo treatment was significantly higher in the 1 placebo-per-day condition $(M=9.27, S E=0.22)$ than the 4 placebos-per-day condition $(M=8.23, S E=0.31), t(58)=2.75, p=.008$. A Pearson's correlation between 
baseline treatment expectancies and subsequent treatment adherence was not significant, $r$ (n $=60)=-.16, p=.21$. Because groups differed in self-reported adherence, number of placebos per day (1 or 4) was included as a control variable in the regression analyses.

Emotional distress: the combination of group and baseline DASS scores accounted for a significant proportion of variance in depression, anxiety, and stress scores at follow-up, $F(2,57)=13.26, p<.001, R^{2}$ adj $=.29$. The addition of baseline expectancies and self-reported adherence added significantly to explained variance, $F(2,55)=9.23, p<.001, R_{\text {change }}^{2}=.17$. Both expectancies and adherence were significant predictors of DASS scores at follow-up, with higher expectancies and higher adherence both predicting lower scores. The addition of the expectancy by adherence interaction term did not add to explained variance, $F(1,54)=$ $0.42, p=.52, R_{\text {change }}^{2}=.004($ see Table 3$)$.

Positive mental wellbeing: the control variables accounted for a significant proportion of variance in WEBWMS scores at follow-up, $F(2,57)=22.88, p<.001, R_{\text {adj }}^{2}=.43$. The addition of expectancies and adherence in the second step of the model explained an additional $12 \%$ of the variance in post-treatment positive mental wellbeing, $F(2,55)=7.76, p$ $=.001$. Higher expectancies and adherence were significant predictors of higher WEBWMS scores. Adding the expectancy by adherence interaction did not explain any additional variance in the outcome measure, $F(1,54)=0.007, p=.93, R_{\text {change }}^{2}=.000$ (see Table 3$)$.

Physical symptoms: together, baseline symptoms and dose were significant predictors of symptoms at follow-up, $F(2,57)=16.94, p<.001, R^{2}$ adj $=.35$. Adding expectancies and adherence accounted for an additional $7.4 \%$ of the variance in physical symptoms, $F(2,55)=$ $3.65, p=.032$. When examining individual predictors, both higher expectancies and adherence predicted fewer symptoms post-treatment. The expectancy by adherence interaction did not explain additional variance, $F(1,54)=2.30, p=.14, R_{\text {change }}^{2}=.02$ (see Table 3). 
Sleep quality: the control variables, again, were significant predictors of posttreatment sleep quality, $F(2,57)=32.78, R_{\text {adj }}^{2}=.52$. However, in the case of sleep quality, the addition of expectancies and adherence in the second step of the model did not explain any additional variance in sleep outcomes, $F(2,55)=0.53, p=.59, R_{\text {change }}^{2}=.01$. Neither expectancies nor adherence predicted post-treatment sleep-quality. Similarly, adding the expectancy by adherence interaction to the model did not predict additional variance in sleep quality, $F(1,54)=1.81, p=.18, R_{\text {change }}^{2}=.02($ see Table 3$)$.

Given that participants who took the open-label placebo reported better sleep quality than those in the no treatment control condition, this result was surprising. To test whether improvements in the other wellbeing outcomes might have contributed to improved sleep quality independent of expectancies or adherence, change scores were calculated (post - pre) for DASS, WEMWBS, and SHC scales. Three separate regression models were run, to test whether changes in these other outcomes predicted sleep quality at follow-up. Again, baseline sleep quality and dose were entered as control variables, and then change scores were entered in the second step of each model. Changes in depression, anxiety, and stress scores $(p=.98)$, positive mental wellbeing scores $(p=.30)$, and symptoms scores $(p=.67)$ did not predict improved sleep quality following placebo treatment.

Control condition: additional exploratory regression analyses were conducted with only participants allocated to the no treatment control condition. These analyses examined the influence of baseline expectancies on each of the wellbeing outcomes, with baseline scores of the respective outcome variables entered as control variables in the first step of the model, and expectancies entered in the second step. This analytic strategy allowed us to assess the possibility that holding positive expectancies at baseline might have been predictive of enhanced wellbeing outcomes even in the absence of placebo treatment. Conversely, participants with positive baseline expectancies might have been disappointed at being 
randomised to the no treatment control condition, and subsequently experienced poorer wellbeing outcomes. The results of these analyses indicate that baseline expectancies in the control group were not significant predictors of subsequent wellbeing outcomes, in either direction (see Table 4).

\section{DISCUSSION}

This study is the first to demonstrate the efficacy of open-label placebo treatments for enhancing wellbeing in healthy participants, specifically in reducing the experience of emotional distress and physical symptoms, and enhancing positive mental wellbeing and sleep quality. In addition, the results provide important insights into the underlying mechanisms of open-label placebo effects. Expectancies prior to treatment, and adherence to the placebo capsules, were significant independent predictors of psychological wellbeing outcomes and physical symptoms. Although 1 placebo-per-day administration was associated with enhanced treatment adherence, no significant differences in wellbeing outcomes were seen between the 1 per-day and 4 per-day open-label placebo-treated participants.

The results with regard to expectancies are generally in line with prior research into 'deceptive' placebo effects, which also identifies the belief that a treatment will be effective as a primary psychological mechanism underlying the effect $[1,16]$. Baseline expectancies predicted improvements in the experience of emotional distress, physical symptoms, and positive emotional wellbeing. Future research should explore the contributors to positive expectancies about open-label placebo treatments - such as personality factors and beliefs about traditional and complementary treatments - and what strategies might be used to enhance perceptions in those who are sceptical in order to facilitate optimal health outcomes. Recent research has shown that positive beliefs about holistic treatments and perceived necessity of a treatment can enhance the placebo effect of an inert cream described as being natural in origin [62]; similar beliefs may also contribute to open-label placebo effects. 
Notably, expectancy ratings in the present study were not particularly high. When asked how well they believed the open-label placebo would work for them, the mean expectancy rating was 4.9 (on a scale from 0 to 10). At best, this represents a moderate belief in the placebo, and likely indicates a degree of uncertainty about how effective the capsules might be in enhancing wellbeing.

Participants' self-reported adherence also significantly predicted the magnitude of placebo effects in psychological wellbeing outcomes, and the experience of physical symptoms. This finding is consistent with previous research demonstrating that patients who were highly adherent to placebos in double-blind clinical trials had better health outcomes [25], and supports the proposition that this benefit may reflect - at least in part - an enhanced placebo effect [26]. To add to the words of former US Surgeon General C. Everett Koop, neither drugs - nor placebos - work in patients who don't take them. Unlike active medical treatments, positive beliefs about the open-label placebo were unrelated to subsequent adherence [63]. Expectancies and adherence had independent effects on open-label placebo effects. All open-label placebos in the current study had brand name labelling. This association with visual information typically accompanying branded active pharmaceuticals may have enhanced treatment expectancies and contributed to the observed outcomes $[50,64]$. Future research could systematically examine the contribution of brand name labelling to open-label placebo effects.

Taking more placebo pills each day did not enhance the open-label placebo effect. This finding is in contrast to research in which participants believe the placebo treatment to be an active drug $[21,22]$. More frequent engagement with pill-taking behaviour did not provide greater benefits. Indeed, in sleep as well as emotional distress and positive mental wellbeing outcomes, there were small but non-significant effects in favour of the 1 placeboper-day treatment, which can potentially be explained by increased adherence in this 
condition. This finding provides evidence that the mechanisms underlying deceptive and open-label placebo effects differ, particularly with regard to what constitutes perceived treatment strength or potency. When patients believe that the treatment they are taking contains an active ingredient, it follows that taking more pills may be indicative of a higher dose of the active ingredient and thus a stronger treatment. It seems that participants in the current study did not conceptualise taking more pills as being indicative of a larger 'dose' of the placebo treatment. However, it may be that the prescribed doses ( 1 versus 4 placebos per day) were not sufficiently different from one another to see an effect; for example, participants may have viewed both treatment regimens as providing a 'high dose' of placebo treatment. Future research investigating how to enhance the perceived strength of open-label placebos would add to evidence-based clinical application.

The present study suggests that open-label placebo administration of four pills per day - as has been done in previous experimental research $[11,13,14]$ - may be unnecessary. In order to test whether higher doses would be associated with greater open-label placebo effects, we compared the effects of single vs multiple dose placebos. More does not appear to be better in this context, but taking the placebo pills as prescribed is important. Making adherence as easy as possible for patients has the potential to further enhance the efficacy of open-label placebo treatments, and simplified dosing regimens of one placebo pill per day may be preferable [23].

Although improvements in sleep quality showed a large open-label placebo effect in comparison to the no treatment control condition, this effect was unrelated to baseline expectancies. Further, changes in the other wellbeing outcomes were unable to account for these findings. These results are surprising, and we propose two possible explanations to account for this. First, it may be that physical symptoms, and negative and positive emotional experiences, are more closely aligned with lay conceptualisations of 'wellbeing' than sleep 
quality. The single item general expectancy measure may have tapped into beliefs about these outcomes preferentially. Future research would benefit from using more specific outcome expectancy assessments. Second, although overall changes in the other wellbeing factors did not predict the magnitude of sleep quality improvements, it may be that initial positive experiences may have contributed to enhanced expectancies for sleep quality, or that improvements in psychological wellbeing directly contributed to sleep outcomes. Assessing expectancies during treatment may contribute to understanding of the development of openlabel placebo effects.

Similarly, improvements in sleep quality were not associated with self-reported adherence to placebo treatment. Adherence to the placebo administration instructions was assessed only at the end of the treatment course. It may be that adherence in this context was more important on a day-by-day basis, i.e. that taking a placebo pill or pills during the day was associated with improved sleep only on that night. Indeed, previous research highlights the large placebo component associated with hypnotic drug treatments [65]. Assessing adherence more regularly throughout the treatment, rather than as a retrospective recall, might offer more insights into the potential contribution of adherence to open-label placebo effects. It is also possible that additional factors that were not assessed in the current study may have contributed to sleep quality outcomes. A number of possible contributors have been put forward by Ballou and colleagues [15], including hope, prediction processing and the attribution of normal variation to treatment effects (leading to actual subsequent improvement), cognitive reappraisal, and aspects of the clinical interaction. Future research would benefit from developing a broader understanding of the factors than can enhance openlabel placebo effects.

The current study has a number of strengths. The experimenter was blind to the participants' group allocation during the baseline assessment, and the follow-up questionnaire 
was conducted online, minimising the possibility of experimenter bias or demand characteristics influencing the results. In addition, measures including the WEMWBS show low social desirability bias [53]. This does not preclude the possibility that social desirability and demand characteristics could have influenced the self-reported outcomes. However, the use of a computer-administered questionnaire during the study session that was completed in the absence of the experimenter, and a follow-up questionnaire also administered online away from the research environment may have been helpful in addressing these potential limitations; evidence suggests that such methods can result in lower social desirability bias in responses [66].

The minimal experimenter contact, and with a student researcher, provides evidence that open-label placebo effects can occur in the absence of a warm and supportive ongoing relationship with a medical professional [13]. Although these factors are certainly likely to contribute to enhanced outcomes, they do not appear to be necessary to generate open-label placebo effects, at least in healthy participants. In addition, participants were informed that they were taking part in a study of the open-label placebo effect, but they were unaware of the dose manipulation, strengthening these results. Finally, the study sought to assess possible mechanisms contributing to open-label placebo effects: expectancies, dose-response, and adherence. The placebo treatment was administered over a 5-day course, allowing the assessment of adherence. To our knowledge this is the first experimental study to investigate possible mechanisms underlying the open-label placebo effect.

The research is potentially limited by the use of a healthy student sample. However, the paradigm was designed for use in this population, allowing greater experimental control and assessment of underlying mechanisms. Previous research has found open-label placebo effects in clinical samples across a range of age groups and conditions $[10,11,13,14]$, and evidence of open-label placebo effects in healthy participants in the current study adds to our 
understanding of this phenomenon. University students commonly report high levels of distress and health complaints, poor sleep quality, and low levels of positive mental wellbeing [67-70]. At follow-up in the current study, only 59\% of no treatment control participants were in the normal range for depression scores [71], compared to $80 \%$ of openlabel placebo-treated participants. The magnitude of these differences have potential implications for health outcomes; even subclinical levels of depression have been linked with increased risk of mortality [72].

The present study may also have been limited by the use of a single-item self-report measure of treatment adherence, rather than using a structured multi-item questionnaire or the inclusion of a pill count at follow-up. However, many commonly used self-report questionnaires assess aspects of adherence such as choosing to stop taking medication due to adverse effects, which were less applicable for the current study [e.g. 72]. Promisingly, single-item self-rated adherence measures like the one used in this study have been shown to provide estimates of adherence similar to unannounced pill counts [74], and self-reported adherence is a good predictor of clinical health outcomes [75]. In addition, instructions to participants in conjunction with the online follow-up may have encouraged generally honest responding and reduced possible social desirability bias.

Finally, providing all participants with open-label placebo information prior to randomisation may have resulted in disappointment in those allocated to the no treatment control condition, which could have caused a nocebo-like effect [see 76 for a review] whereby group differences seen in the current study were due to control participants experiencing worse outcomes (rather than improvement in placebo-treated participants). However, the wellbeing scores of control group participants did not significantly diminish over time, and positive expectancies in this group did not predict subsequent negative 
outcomes. Future research would be strengthened by providing information about open-label placebos only to placebo-treated participants.

The current study, to our knowledge, is the first to explore the mechanisms underlying open-label placebo effects. Results indicate that both expectancies and adherence contribute to enhanced wellbeing outcomes. In contrast, taking more pills per day did not offer additional benefits. In previous research with clinical populations, patients are typically given two placebo pills to take twice per day [13]. The results of the current study indicate that this approach may not be optimally harnessing adherence-driven mechanisms of the open-label placebo effect, and that simplified dosing regimens and other adherence-enhancing strategies might yield even larger effects than have been evidenced to date. Understanding the factors that contribute to open-label placebo effects will allow for optimal application in clinical care, with the potential for utilising placebo effects without deception to improve patient outcomes. 


\section{REFERENCES}

1. Stewart-Williams S, Podd J: The placebo effect: Dissolving the expectancy versus conditioning debate. Psychol Bull. 2004; 130:324-340.

2. Finniss DG, Kaptchuk T, Miller F, Benedetti F: Biological, clinical, and ethical advances of placebo effects. Lancet. 2010; 375:686-695.

3. Miller F, Colloca L, Kaptchuk T: The placebo effect: illness and interpersonal healing. Perspect Biol Med. 2009; 52:518-539.

4. Bingel U, Lorenz J, Schoell E, Weiller C, Büchel C: Mechanisms of placebo analgesia: rACC recruitment of a subcortical antinociceptive network. Pain. 2006; 120:8-15.

5. Kong J, Gollub R, Rosman I, Webb J, Vangel M, Kirsch I: Brain Activity Associated with Expectancy-Enhanced Placebo Analgesia as Measured by Functional Magnetic Resonance Imaging. J Neurosci. 2006; 26:381-388.

6. Mayberg HS, Silva JA, Brannan SK, et al.: The functional neuroanatomy of the placebo effect. Am J Psychiatry. 2002; 159:728-737.

7. De la Fuente-Fernandez R, Ruth TJ, Sossi V, Schulzer M, Calne DB, Stoessl AJ: Expectation and dopamine release: mechanism of the placebo effect in Parkinson's Disease. Science. 2001; 293:1164-1166.

8. Miller F, Wendler D, Swartzman LC: Deception in research on the placebo effect. PLoS Med. 2005; 2:0853-0859.

9. Wendler D, Miller FG: Deception in the pursuit of science. Arch Intern Med. 2004; 164:597-600.

10. Sandler AD, Bodfish JW: Open-label use of placebos in the treatment of ADHD: A pilot study. Child Care Health Dev. 2008; 34:104-110.

11. Carvalho C, Caetano JM, Cunha L, Rebouta P, Kaptchuk T, Kirsch I: Open-label placebo treatment in chronic low back pain. Pain. 2016; 157:2766-2772.

12. Kam-Hansen S, Jakubowski M, Kelley JM, et al.: Altered placebo and drug labeling changes the outcome of episodic migraine attacks. Sci Transl Med January. 2014; $8: 218-5$.

13. Kaptchuk T, Friedlander E, Kelley JM, et al.: Placebos without deception: a randomized controlled trial in irritable bowel syndrome. PLoS One. 2010; 5:e15591.

14. Kelley JM, Kaptchuk T, Cusin C, Lipkin S, Fava M: Open-label placebo for major depressive disorder: A pilot randomized controlled trial. Psychother Psychosom. 2012; $81: 312-314$. 
15. Ballou S, Kaptchuk TJ, Hirsch W, et al.: Open-label versus double-blind placebo treatment in irritable bowel syndrome: study protocol for a randomized controlled trial. Trials. 2017; 18:1-15.

16. Kirsch I: Specifying nonspecifics: psychological mechanisms of placebo effects. In: Harrington A, editor. The Placebo Effect: An Interdisciplinary Exploration. Cambridge, MA: Harvard University Press, 1997. p. 166-186.

17. Colloca L, Miller F: How placebo responses are formed: a learning perspective. Philos Trans R Soc B. 2011; 366:1859-1869.

18. Colagiuri B, Schenk L, Kessler M, Dorsey S, Colloca L: The placebo effect: From concepts to genes. Neuroscience. 2015; 307:171-190.

19. Schafer SM, Colloca L, Wager T: Conditioned placebo analgesia persists when subjects know they are receiving a placebo. J Pain. 2015; 16:412-420.

20. Montgomery GH, Kirsch I: Classical conditioning and the placebo effect. Pain. 1997; 72:107-113.

21. de Craen AJM, Moerman DE, Heisterkamp SH, Tytgat GNJ, Tijssen JGP, Kleijnen J: Placebo effect in the treatment of duodenal ulcer. Br J Clin Pharmacol. 1999; 48:853860.

22. Blackwell B, Bloomfield SS, Buncher CR: Demonstration to medical students of placebo responses and non-drug factors. Lancet. 1972; 299:1279-1282.

23. van Dulmen S, Sluijs E, van Dijk L, de Ridder D, Heerdink R, Bensing J: Patient adherence to medical treatment: a review of reviews. BMC Heal Serv Res. 2007; 7:55.

24. Granger BB, Swedberg K, Ekman I, et al.: Adherence to candesartan and placebo and outcomes in chronic heart failure in the CHARM programme: Double-blind, randomised, controlled clinical trial. Lancet. 2005; 366:2005-2011.

25. Simpson S, Eurich D, Majumdar S, et al.: A meta-analysis of the association between adherence to drug therapy and mortality. BMJ. 2006; 333:15.

26. Chewning B: The healthy adherer and the placebo effect. BMJ. 2006; 333:18-9.

27. Bartels DJP, Van Laarhoven AIM, Haverkamp EA, et al.: Role of conditioning and verbal suggestion in placebo and nocebo effects on itch. PLoS One. 2014; 9:.

28. Bartley H, Faasse K, Horne R, Petrie K: You Can't Always Get What You Want: The Influence of Choice on Nocebo and Placebo Responding. Ann Behav Med. 2016; 50:445-451.

29. Bingel $\mathrm{U}$, Wanigasekera $\mathrm{V}$, Wiech $\mathrm{K}$, et al.: The effect of treatment expectation on drug efficacy: imaging the analgesic benefit of the opioid remifentanil. Sci Transl 
Med. 2011; 3:70ra14.

30. Faasse K, Grey A, Jordan R, Garland S, Petrie K: Seeing is believing: impact of social modeling on placebo and nocebo responding. Heal Psychol. 2015; 34:880-885.

31. Neukirch N, Colagiuri B: The placebo effect, sleep difficulty, and side effects: a balanced placebo model. J Behav Med. 2015; 38:273-283.

32. Quinn VF, Colagiuri B: Sources of placebo-induced relief from nausea: the role of instruction and conditioning. Psychosom Med. 2016; 78:365-372.

33. World Health Organization: Basic documents: 48th edition. Basic Doc 48th Ed. 2014; .

34. Enck P, Bingel U, Schedlowski M, Rief W: The placebo response in medicine: minimize, maximise or personalize? Nat Rev Drug Discov. 2013; 12:191-204.

35. Petrie K, Weinman J: Patients' perceptions of their illness: The dynamo of volition in health care. Curr Dir Psychol Sci. 2012; 21:60-65.

36. Verbrugge LM, Ascione FJ: Exploring the iceberg: common symptoms and how people care for them. Med Care. 1987; 25:539-569.

37. Javaheri S, Storfer-Isser A, Rosen CL, Redline S: Sleep Quality and Elevated Blood Pressure in Adolescents. Circulation. 2008; 118:1034-1040.

38. Cappuccio FPF, D'Elia L, Strazzullo P, Miller MA: Quantity and Quality of Sleep and Incidence of Type 2 Diabetes A systematic review and meta-analysis. Diabetes Care. 2010; 33:414-420.

39. Clarke DM, Currie KC: Depression, anxiety and their relationship with chronic diseases: a review of the epidemiology, risk and treatment evidence. Med J Aust. 2009; 190:S54-S60.

40. Hemingway H, Marmot M: Clinical Evidence: Psychosocial factors in the etiology and prognosis of coronary heart disease: systematic review of prospective cohort studies. West J Med. 1999; 171:342-50.

41. Golden SH, Williams JE, Ford DE, et al.: Depressive symptoms and the risk of type 2 diabetes: the Atherosclerosis Risk in Communities study. Diabetes Care. 2004; 27:429-35.

42. Faasse K, Petrie K: Stress, Coping and Health. In: International Encyclopedia of the Social \& Behavioral Sciences. Elsevier, 2015. p. 551-555.

43. Steptoe A, Kivimaki M: Stress and cardiovascular disease: an update on current knowledge. Annu Rev Public Health. 2013; 34:337-354.

44. Steptoe A, Wardle J, Marmot M: Positive affect and health-related neuroendocrine, cardiovascular, and inflammatory processes. Proc Natl Acad Sci U S A. 2005; 
102:6508-12.

45. Tugade M, Fredrickson B, Feldman Barrett L: Psychological resilience and positive emotional granularity: examining the benefits of positive emotions on coping and health. J Pers. 2004; 72:1161-1190.

46. Fredrickson BL: Cultivating positive emotions to optimize health and well-being. Prev Treat. 2000; 3:1-25.

47. Pressman SD, Cohen S: Does positive affect influence health? Psychol Bull. 2005; 131:925-971.

48. Salovey P, Rothman a J, Detweiler JB, Steward WT: Emotional states and physical health. Am Psychol. 2000; 55:110-121.

49. Faasse K, Martin LR, Grey A, Gamble G, Petrie KJ: Impact of brand or generic labeling on medication effectiveness and side effects. Heal Psychol. 2016; 35:.

50. Branthwaite A, Cooper P: Analgesic effects of branding in treatment of headaches. $\mathrm{Br}$ Med J. 1981; 282:1576-1578.

51. Lovibond S, Lovibond P: Manual for the Depression Anxiety Stress Scales. 2nd ed. Sydney: Psychology Foundation, 1995.

52. Henry JD, Crawford JR: The short-form version of the Depression Anxiety Stress Scales. Br J Clin Psychol. 2005; 44:227-239.

53. Tennant R, Hiller L, Fishwick R, et al.: The Warwick-Edinburgh Mental Well-being Scale (WEMWBS): development and UK validation. Health Qual Life Outcomes. 2007; 5:63.

54. Eriksen HR, Ihlebaek C, Ursin H: A scoring system for subjective health complaints (SHC). Scand J Public Health. 1999; 27:63-72.

55. Filipkowski KB, Smyth JM, Rutchick AM, et al.: Do healthy people worry? Modern health worries, subjective health complaints, perceived health, and health care utilization. Int J Behav Med. 2010; 17:182-188.

56. Buysse DJ, Reynolds CF, Monk TH, Berman SR, Kupfer DJ: The Pittsburgh Sleep Quality Index (PSQI): A new instrument for psychiatric research and practice. Psychiatry Res. 1989; 28:193-213.

57. Grandner MA, Kripke DF, Yoon I-Y, Youngstedt SD: Criterion validity of the Pittsburgh Sleep Quality Index: Investigation in a non-clinical sample. Sleep Biol Rhythms. 2006; 4:129-139.

58. Carpenter JS, Andrykowski MA: Psychometric evaluation of the Pittsburgh Sleep Quality Index. J Psychosom Res. 1998; 45:5-13. 
59. Simpson RJ, Mendys P: The effects of adherence and persistence on clinical outcomes in patients treated with statins: A systematic review. J Clin Lipidol. 2010; 4:462-471.

60. Ho PM, Bryson CL, Rumsfeld JS: Medication adherence: Its importance in cardiovascular outcomes. Circulation. 2009; 119:3028-3035.

61. Vickers AJ, Altman DG: Analysing controlled trials with baseline and follow up measurements. BMJ. 2001; 323:1123-1124.

62. Watkinson A, Chapman SCE, Horne R: Beliefs about pharmaceutical medicines and natural remedies explain individual variation in placebo analgesia. J Pain. 2017; .

63. Horne R, Chapman SC, Parham R, Freemantle N, Forbes A, Cooper V: Understanding patients' adherence-related beliefs about medicines prescribed for long-term conditions: a meta-analytic review of the Necessity-Concerns Framework. PLoS One. 2013; 8:e80633.

64. Faasse K, Martin LR, Grey A, Gamble G, Petrie KJ: Impact of brand or generic labeling on medication effectiveness and side effects. Heal Psychol. 2016; 35:187190.

65. Winkler A, Rief W: Effect of Placebo Conditions on Polysomnographic Parameters in Primary Insomnia: A Meta-Analysis. Sleep. 2015; 38:925-31.

66. Richman WL, Kiesler S, Weisband S, Drasgow F: A meta-analytic study of social desirability distortion in computer-administered questionnaires, traditional questionnaires, and interviews. J Appl Psychol. 1999; 84:754-775.

67. Bayram N, Bilgel N: The prevalence and socio-demographic correlations of depression, anxiety and stress among a group of university students. Soc Psychiatry Psychiatr Epidemiol. 2008; 43:667-672.

68. Jensen DR: Understanding sleep disorders in a college student population. J Coll Couns. 2003; 6:25-34.

69. Edwards KJ, Hershberger PJ, Russell RK, Markert RJ: Stress, negative social exchange, and health symptoms in university students. J Am Coll Health. 2001; 50:7579.

70. Stallman H: Psychological distress in university students: A comparison with general population data. Aust Psychol. 2010; 45:249-257.

71. Crawford J., Henry JD: The depression, anxiety and stress scales (DASS): normative data and latent structure in a large non-clinical sample. Br J Clin Psychol. 2003; 42:111-131.

72. Cuijpers P, Smit F: Excess mortality in depression: A meta-analysis of community 
studies. J Affect Disord. 2002; 72:227-236.

73. Morisky DE, Green LW, Levine DM: Concurrent and predictive validity of a selfreported measure of medication adherence. Med Care. 1986; 24:67-74.

74. Kalichman SC, Amaral CM, Swetzes C, et al.: A simple single-item rating scale to measure medication adherence: further evidence for convergent validity. J Int Assoc Physicians AIDS Care (Chic). 2009; 8:367-74.

75. Stirratt MJ, Dunbar-Jacob J, Crane HM, et al.: Self-report measures of medication adherence behavior: recommendations on optimal use. Transl Behav Med. 2015; $5: 470-482$.

76. Faasse K, Petrie K: The nocebo effect: patient expectations and medication side effects. Postgrad Med J. 2013; 89:540-6. 
Table 1. Baseline between-group comparisons on demographic and wellbeing measures.

\begin{tabular}{|c|c|c|c|c|c|}
\hline & $\begin{array}{c}\text { Control } \\
\% / \mathrm{M}(\mathrm{SD})\end{array}$ & $\begin{array}{c}1 \text { placebo-per-day } \\
\% / \mathrm{M}(\mathrm{SD})\end{array}$ & $\begin{array}{c}4 \text { placebos-per-day } \\
\% / \mathrm{M}(\mathrm{SD})\end{array}$ & $\chi^{2} / \mathrm{F}$ & $p$-value \\
\hline Gender (\% female) & $80 \%$ & $77 \%$ & $84 \%$ & 0.42 & .81 \\
\hline Age & $18.9(2.00)$ & $20.0(4.86)$ & $20.5(3.87)$ & 1.26 & .29 \\
\hline Emotional distress (DASS) & $12.73(9.04)$ & $16.09(12.28)$ & $14.97(8.41)$ & 0.88 & .42 \\
\hline Positive mental wellbeing (WEMWBS) & $47.70(8.95)$ & $47.71(10.76)$ & $46.90(9.31)$ & 0.07 & .93 \\
\hline Physical symptoms (SHC) & $16.43(8.88)$ & $20.68(12.31)$ & $19.58(10.09)$ & 1.33 & .27 \\
\hline Sleep quality (PSQI) & $7.20(2.54)$ & $7.03(2.95)$ & $7.25(2.72)$ & 0.06 & .95 \\
\hline Expectancies & $4.57(2.33)$ & $5.13(2.43)$ & $5.00(2.21)$ & 0.49 & .62 \\
\hline
\end{tabular}


Table 2. Mean (SE; 95\%CI) between-group post-treatment scores for each outcome variable, adjusted for the corresponding baseline scores.

\begin{tabular}{lccc}
\hline & $\begin{array}{c}\text { Control } \\
\text { M (SE; 95\%CI })\end{array}$ & $\begin{array}{c}\text { 1 placebo-per-day } \\
\text { M (SE; 95\%CI })\end{array}$ & $\begin{array}{c}4 \text { placebos-per-day } \\
\text { M (SE; 95\%CI) }\end{array}$ \\
\hline Emotional distress (DASS) & $13.47(1.24 ; 11.01$ to 15.93$)$ & $6.97(1.17 ; 4.64$ to 9.30) & $8.48(1.15 ; 6.57$ to 11.13$)$ \\
Positive mental wellbeing (WEMWBS) & $45.07(1.52 ; 42.05$ to 48.09$)$ & $52.44(1.44 ; 49.48$ to 55.30$)$ & $49.39(1.42 ; 46.57$ to 52.20$)$ \\
Physical symptoms (SHC) & $16.93(2.28 ; 12.39$ to 21.46$)$ & $10.80(2.12 ; 6.59$ to 15.01$)$ & $11.16(2.07 ; 7.03$ to 15.28$)$ \\
Sleep quality (PSQI) & $7.09(0.35 ; 6.39$ to 7.79$)$ & $5.22(0.33 ; 4.56$ to 5.87$)$ & $5.83(0.32 ; 5.19$ to 6.47$)$ \\
\hline
\end{tabular}


Table 3. Standardized beta, t-, and p-values from the full regression model (for placebotreated participants only) for each outcome variable.

\begin{tabular}{|c|c|c|c|}
\hline & $\beta$ & $t$ & $p$-value \\
\hline \multicolumn{4}{|l|}{ Depression, Anxiety, and Stress } \\
\hline Baseline DASS & 0.59 & 6.04 & $<.001$ \\
\hline Dose & 0.02 & 0.23 & .86 \\
\hline Expectancy & -0.36 & -3.41 & .001 \\
\hline Adherence & -0.36 & -3.24 & .002 \\
\hline Expectancy $x$ Adherence & 0.07 & 0.65 & .52 \\
\hline \multicolumn{4}{|l|}{ Positive Mental Wellbeing } \\
\hline Baseline WEMWBS & 0.63 & 7.02 & $<.001$ \\
\hline Dose & -0.06 & -0.67 & .51 \\
\hline Expectancy & 0.31 & 3.18 & .002 \\
\hline Adherence & 0.25 & 2.46 & .017 \\
\hline Expectancy $x$ Adherence & -0.01 & -0.08 & .93 \\
\hline \multicolumn{4}{|l|}{ Symptoms Score } \\
\hline Baseline SHC & 0.67 & 6.47 & $<.001$ \\
\hline Dose & -0.04 & -0.42 & .68 \\
\hline Expectancy & -0.29 & -2.60 & .012 \\
\hline Adherence & -0.27 & -2.39 & .020 \\
\hline Expectancy $x$ Adherence & 0.17 & 1.52 & .14 \\
\hline \multicolumn{4}{|l|}{ Sleep Quality } \\
\hline Baseline PSQI & 0.70 & 7.72 & $<.001$ \\
\hline Dose & 0.14 & 1.49 & .14 \\
\hline Expectancy & 0.01 & 0.13 & .90 \\
\hline Adherence & 0.13 & 1.25 & .22 \\
\hline Expectancy $x$ Adherence & -0.14 & -1.35 & .18 \\
\hline
\end{tabular}


Table 4. Standardized beta, $\mathrm{t}-$, and $\mathrm{p}$-values from the control group only regression model for each outcome variable.

\begin{tabular}{lccc}
\hline & $\beta$ & $t$ & $p$-value \\
\hline Depression, Anxiety, and Stress & & & $<.001$ \\
$\quad$ Baseline DASS & 0.82 & 6.94 & .66 \\
$\quad$ Expectancy & 0.05 & 0.45 & \\
Positive Mental Wellbeing & & & $<.001$ \\
Baseline WEMWBS & 0.84 & 7.01 & .28 \\
Expectancy & -0.13 & -1.10 & .30 \\
Symptoms Score & & & .65 \\
Baseline SHC & 0.22 & 1.06 & \\
Expectancy & -0.10 & -0.47 & .001 \\
Sleep Quality & & & .16 \\
Baseline PSQI & 0.91 & 9.12 & \\
Expectancy & 0.14 & 1.44 & \\
\hline
\end{tabular}



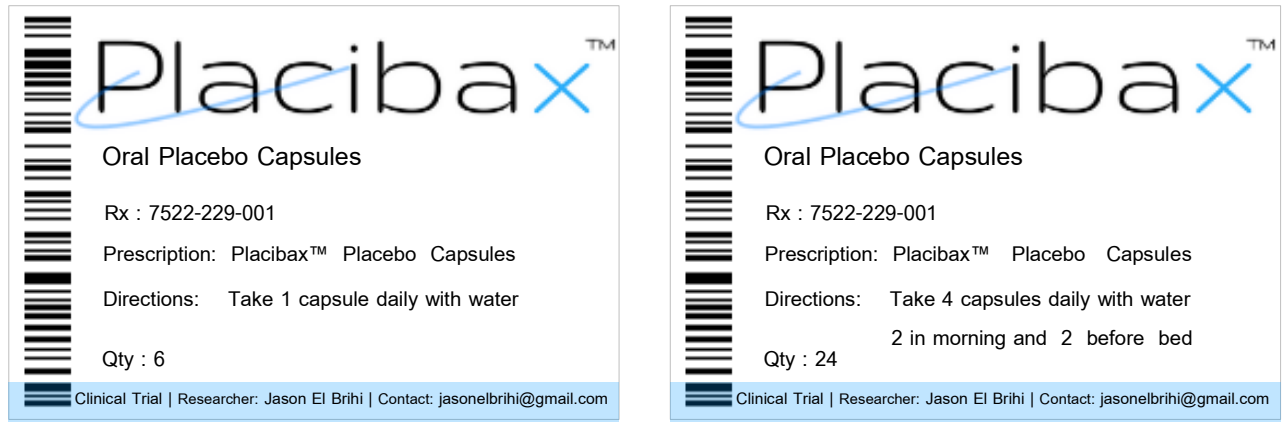

Figure 1. Labels for the 1 per-day (left) and 4 per-day (right) open-label placebo conditions. 

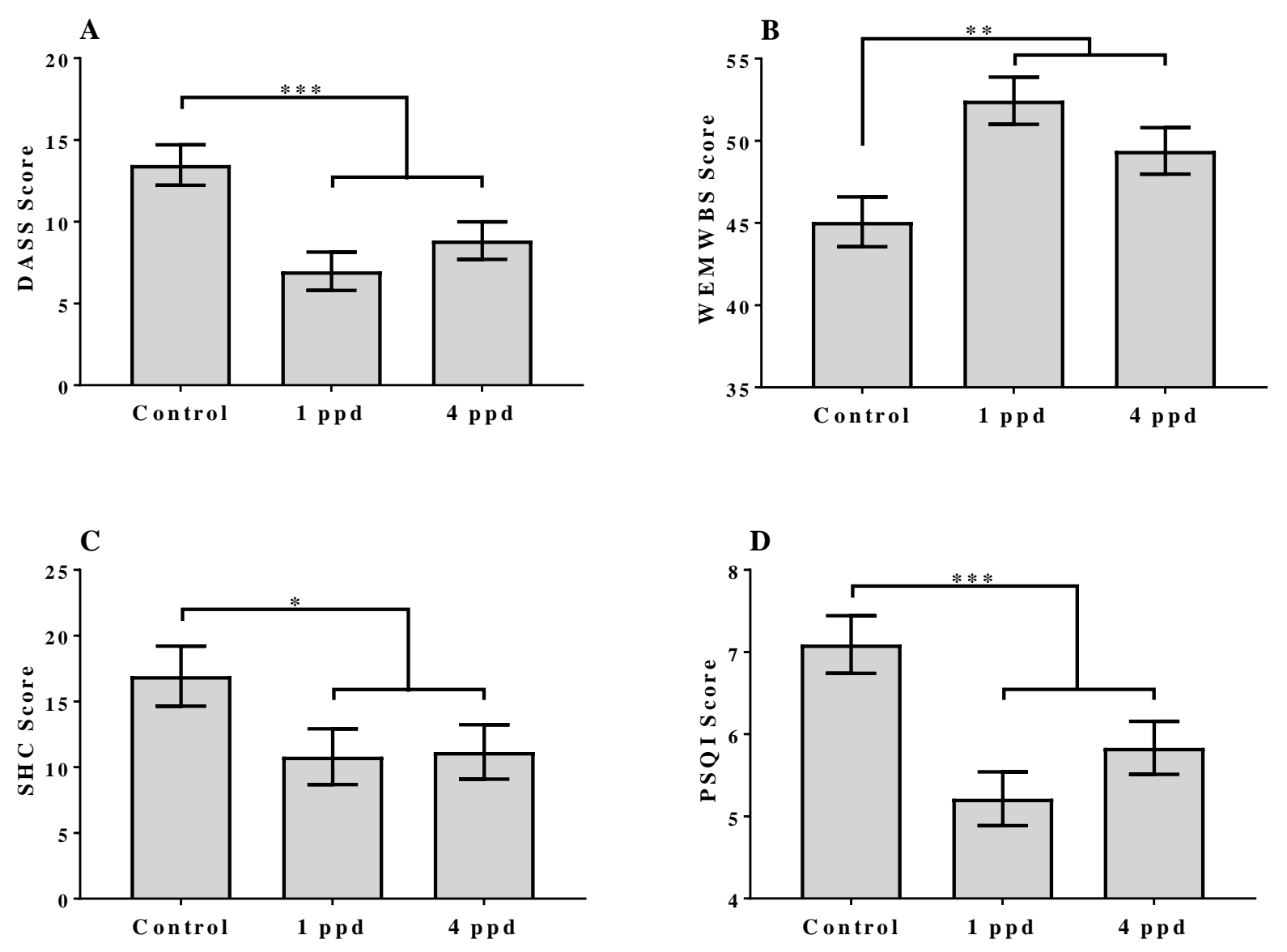

Figure 2. Bar graphs showing baseline-adjusted mean (SE) scores for no treatment control, 1 placebo-per-day (ppd), and 4 placebos-per-day (ppd) conditions across wellbeing outcomes of A) emotional distress, B) positive mental wellbeing, C) physical symptoms, and D) sleep quality. $* * * p<.001 ; * * p<.01 ; * p<.05$ 